\title{
Silicon detector for the Saline Soil Degree in ETM Image
}

\author{
Tao $\mathrm{Chi}^{1}$, Bingchun $\mathrm{Li}^{2}$ \\ ${ }^{1}$ College of Information Technology, Shanghai Ocean University, Shanghai, 201306, China \\ ${ }^{2}$ Department of Information Engineering Technology, Kashi Teacher College, Xinjiang, 844006, \\ China
}

Keywords: infrared radiation, silicon detector, sensor pre-processing, ETM-technology, multichannel signal processing.

\begin{abstract}
A silicon photoconductor apparatus having improved efficiency in infrared radiation detection by sandblasting the reflective surface of the apparatus and thereby creating a near-infinite number of reflecting angles and a corresponding multiplication of quantum energy yield. This article describes its architecture, including its design, implementation, and performance. The novel method, a combination of surface barrier and planar processes, has resulted in detectors with excellent quality and long term operation stability. A current density of down to $1 \mathrm{nA} / \mathrm{cm} 2$ was reached. Studies of radiation damage produced by $14 \mathrm{MeV}$ neutrons, $19 \mathrm{MeV}$ protons and $17 \mathrm{keV}$ $\mathrm{X}$-rays were performed and the resulting current increase, impurity removal and charge collection deficiency investigated. Long term storage and short term heat treatments showed appreciable annealing effects by up to $83 \%$. In addition, we establish a testing system that can measure the soil salinization. Finally, we describe the reflection characteristics of visible, infrared and microwave in soil, which can be gotten by combining the spectrogram with the TM data.
\end{abstract}

\section{Introduction}

The saline soil is a worldwide problem, while the research on dynamic aspect of the saline soil mainly focuses on the monitoring, assessment and forecasting techniques. The traditional method of the saline soil monitoring is the laboratory assay by extracting the soluble salt from the waterlogged soil [2]. This method has many disadvantages, such as complex operating procedure, poor real-time and high detection cost. Some achievement of the research on the saline soil information has been got at home and abroad $[4,5,6]$. At present, the information extraction of the saline soil is mainly based on the spectral response characteristics, which simulates and predicts the saline soil by combining the RS, GIS with expert system [7]. Because the saline soil is seriously mixed with the other soil features, the spectral response characteristics can not accurately predict the quantitative degree of the saline soil, only the qualitative analysis can be done with the results [8]. Therefore, accurate and automatic extraction of the saline soil has much important implication for the monitoring and the research on the dynamic aspect of the saline soil. This study adopts the multiinformation acquisition technology by using the multi-sensor array. Based on this technology, the related portable equipment aiming to quickly monitor the saline soil can be developed. Then the saline soil conditions can be quickly and conveniently gotten, and the saline soil degree can be identified. The result of this research can be applied to model the precise agriculture facilities and regulate the greenhouse.

From the literatures at home and abroad, soil salinity can be on behalf the soil conductivity. Many elements of the soil have a diagnostic absorption feature in the solar spectral range (0.3$2.5 \mu \mathrm{m})$. The researchers have done much research on the relation between the soil properties and laboratory spectral reflectance, and successfully predicted the soil composition by using the technology of reflectance spectroscopy $[9,10]$.

\section{The design of multi-sensor detector}

Multi-sensor array can be divided into two parts, the one part of sensors device is used to detect the soil conductivity, the other is used to monitor the spectral image. The core of the multi-sensor 
detector is operating at wavelength of $1.02 \mu$ and the application of efficient and powerful lasers at wavelengths of 1.02 microns. In typical silicon photodiodes, for detecting $1.02 \mu$ radiation, the requirements for high speed and high sensitivity are mutually exclusive. Since the electrode separation is any greater than $0.1 \mathrm{~mm}$, the minority carrier transit time will be greater than $1 \mathrm{nsec}$. This problem can be solved by allowing the incident light beam to make multiple passes between the electrodes. The optical path length can then be extended to several millimeters, as required for complete absorption, while the electrode separation remains less than $0.1 \mathrm{~mm}$, as required for nanosecond response time. In a typical photodiode geometry, one ohmic contact and one rectifying contact are formed on the two opposite surfaces of a base wafer, and the wafer thickness determines the electrode separation. The objective of the multiple reflection design is to allow all $1.02 \mu$ radiation to enter the detector front surface and from the back detector surface so that no $1.02 \mu$ radiation can exit. Total internal reflection at the back detector surface is well suited for light trapping of $1.02 \mu$ radiation because the relatively large dielectric constant of silicon leads to a critical angle of $15 \circ$ for total internal reflection. Detectors utilizing total internal reflection have been constructed in silicon. Silicon photodiodes are widely used in the detection of visible and near infrared radiation. They can be readily designed to have high quantum efficiency for wavelengths from $5000 \mathrm{~A}$. to $7500 \mathrm{~A}$. and fast response time. The main drawback with these typical photodiodes for detecting $1.02 \mu$ micron radiation is that the requirements for high speed and high sensitivity are mutually exclusive.

Since the absorption coefficient is only $25 \mathrm{~cm}-1$, a $1 \mathrm{~mm}$ path length is required to absorb 91 percent of the incident $1.02 \mu$ radiation.

The sensors device monitoring the spectral image consists of LED source, CCD detector, TM image fusion and classification processing software. The light from the light source forms the punctuate light through the lens and then incident to the surface. The photon into the soil would escape from the surface after scattering, and can be collected by the detector and forms into scattering images, finally the spectral image can be formed by the filtering chip.

The multi-sensors for detecting soil conductivity

The sensors device detecting the soil conductivity consists of transmitter and receiver. The transmitter is a small transmitting coil, which can produce a dynamic primary magnetic field according to change of the soil depth along with time. This magnetic field can then produce a little weak AC induction current in the earth, which induces the secondary magnetic field. Then the receiving coil of the receiver receives the secondary electromagnetic signal produced by the soil conduction current. The received electrical signal, which is proportional to the soil conductivity, can appear as the soil conductivity after computing and processing. The ratio of the primary and secondary magnetic field is usually in a linear relationship with the soil conductivity.

The detected signals are rather weak, so it is necessary to magnify these weak signal, the collected signals have much noise resulting from the magnification. Owing to the noise, the special signal processing circuit is specially designed to magnify signals and decrease noise.

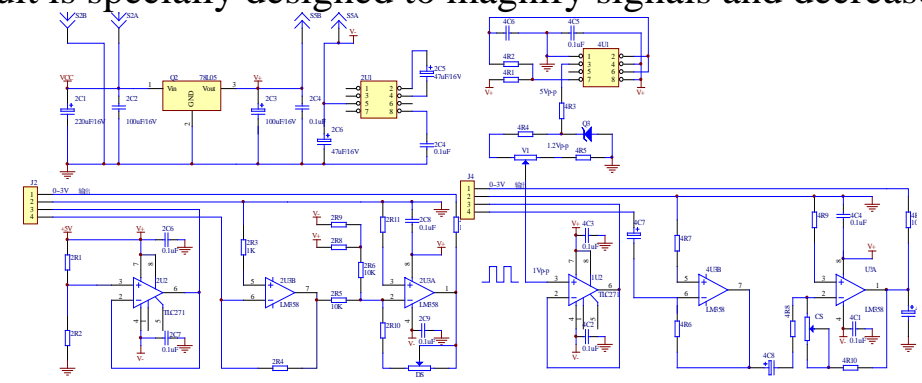

Fig.1: The special processing circuit of detecting the soil conductivity

In particular, electromagnetic induction devices are sensitive to metal, so it is important to minimize the metal objects within the induction range, the sub components are the PVC bracket and some non-metallic products which are used to connect, fix and adjust the equipments.

\section{The multi-sensors for monitoring TM image}

The sensors device monitoring the spectral image consists of LED source, CCD detector, TM 
image fusion and classification processing software. The light from the light source forms the punctuate light through the lens and then incident to the surface. The photon into the soil would escape from the surface after scattering, and can be collected by the detector and forms into scattering images, finally the spectral image can be formed by the filtering chip.

\section{A. The selection of light source}

The following elements can be chosen as tracers for a specific source type: $\mathrm{Cu}$ for industrial heavy oil combustion, $\mathrm{Pb}$ for traffic, $\mathrm{Cd}$ and $\mathrm{Zn}$ for refuse incineration plants and $\mathrm{Cd}$ and $\mathrm{Cu}$ for the cement industry. Size-resolved elemental concentrations will make it easier to distinguish between sources Bratanow et al [1]. Therefore similar industrial plants could emit different types of dusts and then different types of metal pollutant. In this paper we consider a particular location, situated near two cement plants, that is characterized by dust pollution that does not exceed the law limits and we determine which substances are really present in the dusts.

Two analysis wavelength can be determined according to the study of spectroscopy, they are water absorption peak(760nm) and electrolytic salt absorption peak $(810 \mathrm{~nm})$. The high-intensity light-emitting diode LED is used as the single-wavelength light source. The light intensity of LED is strong enough to be used as the light source of the portable wavelength equipment.

B. The selection of CCD detector

In order to improve the detection accuracy, the exposed area detecting the light is expected to be large. At the same time, the weak signals require the higher detector sensitivity. Since the instrument developed in this topic targets at the low-cost portable instrument, so the moderate costeffective devices are preferred. The low-cost silicon testing is selected as the detector and can be divided into domestic and imported models. The exposed area of domestic detector is bigger than that of the imported ones, but the dark current of the domestic detector is stronger than that of the imported ones. So four imported detectors are adopted, the larger exposed area and higher sensitivity can be gotten by parallel arranged the four detectors.

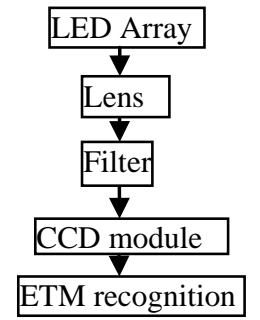

Fig.2: Example of ETM recognition

\section{TM image fusion and classification}

It is generally believed that the soil reflectivity ranges between the water bodies and vegetation, it means that the soil reflectivity is more than water bodies but less than vegetation. In the typical spectral curve of the soil features, the reflectivity of salt-smarsh soil and general soil is more than the other soil features when the wavelength ranges between 0.35-0.65 $\mu \mathrm{m}$ (visible light band). The reflectivity of the vegetation is more than that of soil when the wavelength is above $0.65 \mu \mathrm{m}$ (nearinfrared band). The reflectivity of the soil is more than that of the vegetation in the far-infrared band. This phenomenon mentioned above mainly results from the physicochemical properties of soil, water dynamics and leaf reflectance characteristics of vegetation. Dwivedi R.S. did many pilot studies of best band combination about the saline soil. If the amount of information is uniquely adopted to measure the reflectivity, TM data combination of $1,3,5$ bands contains the most information, but the information accuracy extracted from the saline soil is not proportional to the size of the amount of information. Accordingly, synthetic program of 1, 3, 5-band is adopted as the basis image, because this synthetic program can best reflect the tone and texture of the spectral characteristics of the saline soil, such as color and texture. The TM data is adopted as the basis image of the saline soil in this topic.

\section{The pretreatment of TM image}

The mode that the user inputs coordinate is adopted to geometric correct the TM image in the 
study area, the corresponding ground control points can be selected in the diagram. When calibrated, the corresponding relationship between the map coordinate $(\mathrm{x}, \mathrm{y})$ and the image data coordinate (x, $y)$ is: $X=F(x, y), Y=G(x, y)$. The specific methods are as follows. In the TM image, 20 obvious features, such as: cross-roads and cross-road-trench, can be selected as the ground control points.

The model of polynomial calibration and the model of the most close distance re-sampling are adopted to geometric correct the original image. The average of RMS (Root Mean Square error) error is 0.25 after ENVI processing, which well meets the requirement of controlling the error within a pixel. In the image processing, the bilinear interpolation is adopted to re-sample the gray value of pixel to avoid the brightness discontinuity of the nearest neighbor method.

\section{The method of image fusion}

In order to better improve the spectral and spatial resolution of fusion image and get better fusion image, it is critical to adopt the most suitable method for the study area image. At present, there are many methods of disposing the remote sensing image, it mainly includes LAB transformation, HIS transformation, Linear recombination and product operation, Brovey transformation, Wavelet transformation, High-pass filter transformation and Principal component analysis and so on.

The method of principal component analysis is adopted to study the TM image in this paper. The first principal component reflects the level of pixel brightness, which cannot clearly distinguish the types of soil features. The second principal component reflects the level of chlorophyll content of the pixel. The fourth principal component reflects the information of the water bodies, the contributions of the other two principal components are rather little and it is difficult to analyze their physical meaning in general. The third principal component basically reflects the infrared radiation level, which if corresponding to the water depth and the salted land information. So PC3 is chosen as a characteristic variable to identify the salted land information.

\section{The method of classification}

Due to the presence of image speckle noise, the targets in feature space correspond to complex model. The traditional classification methods (such as the maximum likelihood method, the minimum distance method) do badly in classifying the TM images. This paper adopts the Support Vector Machine (Support Vector Machine, SVM) classification, which is high in accuracy and stability.

Support Vector Machine is a kind of machine learning method proposed by Vapnik and the others during the 1990s, which is a minimization criterion realizing the structural risk. SVM is a universal machine learning method, which is effective in solving pattern recognition and function estimation problems. The study of Vapnik and the others shows that the performance of SVM has little relationship with the selected type of kernel function, while the parameters of kernel function are the main factors that affect the SVM performance. By experimental study, Sigmoid method is feasible to classify the TM image of the saline soil.

\section{Modeling SVM with ETM data}

A spectral mixture model is a physically based model in which a mixed spectrum is modeled as a combination of pure spectra, called end-members. Fractional surface cover of live-photosynthetic green plants, senescent photosynthetic or non-photosynthetic vegetation, water and bare soil were estimated in each ETM image pixel using spectral mixture analysis. The spectral mixture analysis provided estimates of live-photosynthetic vegetation, non-photosynthetic vegetation, water and bare soil covers within each image pixel, along with statistical uncertainty estimates for each cover-type.

$$
\rho(\lambda)_{\text {pixel }}=\sum_{m=1}^{n}\left[C_{m} \times \rho_{m}(\lambda)\right]
$$

We used four spectral end-member to decompose each image pixel using the following equation $\rho(\lambda)_{\text {pixel }}=\left[C_{\text {Water }} \times \rho_{\text {Water }}(\lambda)+C_{\text {soil }} \times \rho_{\text {soil }}(\lambda)+C_{\text {NPV }} \times \rho_{\text {NPV }}(\lambda)+C_{\text {Plant }} \times \rho_{\text {Plant }}(\lambda)\right]+\varphi$

And

$$
\sum_{m=1}^{n} C_{m, \lambda}=1 \quad 0 \leq C_{m, \lambda} \leq 1
$$

Where $\rho_{m}(\lambda)$ is the reflectance of each land-cover end-member at wavelength $\lambda$, and $\varphi$ is a 
residual or error term. The sub-pixel cover fractions $C_{m}$ are Water, NPV, bare soil and plants. The accuracy of the estimated sub-pixel cover fractions $\left(C_{\text {Plant }}, C_{N P V}, C_{\text {soil }}\right)$ relies heavily upon the selection of end-member reflectance $\left(\rho_{\text {Plant }}, \rho_{N P V}, \rho_{\text {soil }}, \rho_{\text {Water }}\right)$, thus we developed reflectance bundles using ETM images together with ground observation sample to obtain sub-pixel cover fractions from radiometric matched images (Philip and Dar, 2003). Therefore, equation (4) can be used to estimate canopy maximum possible light-use efficiency:

$$
\varepsilon=C_{\text {Plant }} \times \sigma
$$

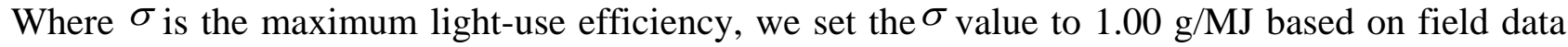
(Running, 2009).

\section{Experimental results}

\section{Unmixed results from ETM images}

The spatial distributions of sub-pixel fractional covers of end-members in 2009 are shown in Fig 3. The main components of photosynthetic plants are the riparian forest and salt meadow and other oasis crops, such as cotton, maize, wheat, etc.

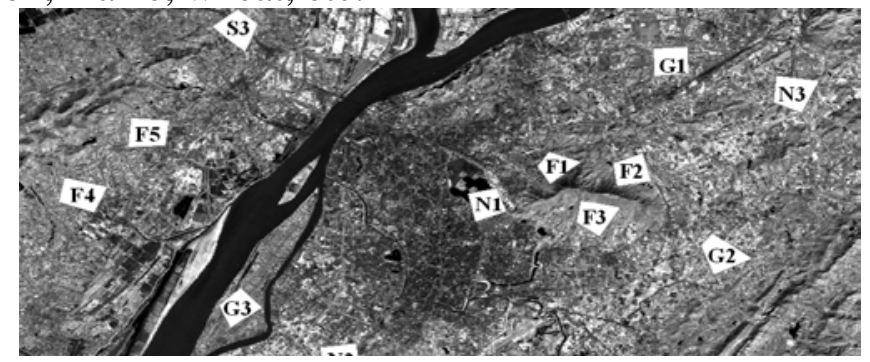

Fig.3: Band 4 of the ETM image (F:Water; N:NPV; S:Soil; G:Greenplants)

\section{Validation of SVM modelling results}

At the landscape scale, the opportunity exists to combine remote sensing data with spatially distributed, process-based biogeochemistry models to examine variation in ecosystem processes such as SVM as a function of land cover type, canopy attributes, and/or location along environmental gradients. There process models can be validated against direct measurements made with ground-based SVM sampling and data from literatures. We use field measurement data to validate our modeled SVM from Database. The total SVM simulated by the model compared relatively well with the direct observations. The simulated SVM was the highest in oasis agriculture and marsh vegetation, intermediate in most forested land and the lowest in field plants (fig. 3). Crops had a relatively low SVM.

\section{Conclusion and discussion}

The present invention describes an apparatus which achieves the same desired result, multiple internal reflection, with none of the attendant limitations of the dimple technique. The reflective surfaces are sandblasted over any desired area in a very short time with the simplest of equipment, forming, in effect, a myriad of dimples. This approach could be used with any crystalline photodetector to enhance the response to wavelengths not absorbed in typical geometries, as long as the increased absorption will result in additional photo-excited carriers. Thus a silicon photo-conductor apparatus is provided which will cause multiple reflections of light, incident to its front surface, within the photo-conductor device produces both the high sensitivity and high speed required to detect $1.02 \mu$ radiation.

This paper establishes a testing system that can measure the saline soil. Silicon conductor method is applied to quantitative analyze the saline soil.

Based on the reflection characteristics of visible, infrared and microwave in soil, the image can be gotten by combining the spectrogram with the TM data. This image is rich in soil texture information, and can be used to qualitatively or quantitatively analyzed the degree of the saline soil. 
It can be seen from the experiments that the method in the paper, which combines the method of electromagnetic properties and the method of spectra properties, is feasible to comprehensively evaluate the degree of saline soil.

\section{Acknowledgements}

This work was supported by Shanghai Municipal Natural Science Foundation.

\section{References}

[1] McCulloch W S, Pitts W. A Logical Calculus of the Ideas Immanent in Nervous Active. Bulletin of Mathematical Btophysics,1943,5:115-133)

[2] David C Hyland, et al. Neural Network System Identification for Improved Noise Rejection. International Journal of Control, 1997,68(2):233-258.

[3] Horton M P. Real-time Identification of Missile Aerodynamics Using a Linearised Kalman Filter Aided by an Artificial Neural Network. IEE Proc. -Control Theory Appl., 1997,144(4):299-308.

[4] Anuradha M Annaswamy, Ssu-Hsin Yu. Adaptive Neural Networks: A New Approach to Prameter Estimation. IEEE Trans. On Neural Netwoks, 1996,7(4):907-918.

[5] Kumpati S. Narendua, Snehasis Mukhopadhyay. Adoptive Control Using Neural Networks and Approximate Models. IEEE Trans. On Neural Networks,1997,8(3):475-485.

[6] Lightbody G, Prof. Irwin G W. Direct Neural Model Reference Adaptive Control. IEE Proc. Control Theory Appl.,1995,142(1):31-43.

[7] Zadeh L A, Fuzzy Sets. Information and Control.1965,8:338-353.

[8] Mamdani E H. Applications of Fuzzy Algorithms for Control of Simple Dynamic Plant. Proc. IEEE,1974,121(21):1585-1588:

[9] Jamshidi M,et al. Fuzzy Logic and Control. Englewood Cliffs,NJ:Prentice Hall,1993

[10] Kandel A, Langholz G, (Eds). Fuzzy control systems. Boca Raton, FL.:CRC Press,1994. 